\title{
EXPANSÃO RÁPIDA DA MAXILA NÃO CIRÚRGICA E CIRÚRGICA: REVISÃO DE LITERATURA
}

\section{EXPANSION OF QUICK JAW SURGICAL AND NON SURGICAL: LITERATURE REVIEW}

\author{
Tiago Estevam de Almeida* \\ Jesus Saavedra* \\ Marcela Pavlovsky \\ José Antônio Scrocco \\ Maurício Gomes dos Santos \\ Carolina Gioia Monteiro
}

\section{RESUMO}

As deficiências transversais são deformidades dentofaciais relativamente comuns na clínica odontológica. Os pacientes nessas condições podem apresentar apinhamentos dentários, discrepâncias mandibulares, mobilidade dos dentes, perdas múltiplas dentárias, recessões gengivais, dificuldades respiratórias, sinusites de repetição, além de serem respiradores bucais. Nesse sentido, as técnicas de expansão rápida da maxila não cirúrgica (ERM) e cirúrgica são utilizadas, a fim de corrigir por meio da disjunção palatina o desenvolvimento maxilar. A ERM está contraindicada em pacientes com maturação óssea completa e, para esses casos, se indica a expansão rápida cirúrgica de maxila (ERMA), sendo esta preferível com expansor Hyrax. A técnica de expansão de maxila utilizando Hass mostra-se satisfatória devido à distribuição da tração ocorrer no centro da maxila e ser a técnica cirúrgica mais condizente com os parâmetros atuais e a menos invasiva com a utilização do expansor Hyrax considerando-se a higienização do paciente.

DESCRITORES: Técnica de expansão palatina

\section{ABSTRACT}

The transverse deficiencies are relatively common dentofacial deformities in the dental clinic. Patients in these conditions may have crowding teeth, jaw discrepancies, mobility of teeth, multiple teeth loss, gum recession, breathing difficulties, recurrent sinusitis, and are mouth breathers. In this sense, the techniques of rapid maxillary expansion nonsurgical (ERM) and surgery are used to correct rapid palatal expansion by maxillary development. The ERM is contraindicated in patients with complete bone maturation, and for these cases indicated the rapid expansion of jaw surgery (ERMA), which is preferable with Hyrax expander. The technique of maxillary expansion using Hass proves to be satisfactory due to the distribution of traction to be the center of the jaw and the surgical technique more suited to today's standards, is the least invasive with the use of the Hyrax expander considering the hygiene of the patient.

DESCRIPTORS: Palatal expansion technique

\footnotetext{
* Mestre em Cirurgia Bucomaxilofacial pela USP. Residência em Cirurgia Bucomaxilofacial pelo HSPE/FMO-SP, Especialista em Implantodontia pela CIODONTO. Cirurgião Bucomaxilofacial do HMU de Guarulhos. Aluno do curso de graduação em Medicina da UNICID-SP.

** Especialista em Cirurgia Bucomaxilofacial com Residência no Hospital do Tatuapé-SP e Cirurgião Bucomaxilofacial do PA São João da Santa Casa de Misericórdia de São Paulo.

*** Cirugiã Dentista Especialista em Ortodontia pela Universidade Cruzeiro do Sul-SP.

**** Graduado em Odontologia pela UNIP e Aluno do curso de graduação em Medicina da UNICID-SP.

***** Residência em Cirurgia Bucomaxilofacial pelo HSPE/FMO-SP, Cirurgião Bucomaxilofacial do HMU de Guarulhos e do Hospital Heliópolis-SP. ****** Aluna do curso de graduação em Medicina da UNICID-SP.
} 
ALMEIDA TE

SAAVEDRA J

PAVLOVSKY M

SCROCCO JA

SANTOS MG

MONTEIRO CG

EXPANSÃO RÁPIDA

DA MAXILA NÃO

CIRÚRGICA E

CIRÚRGICA :

REVISÃO DE

LITERATURA

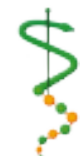

REV, ODONTOL.

UNIV, C I D. SÃO

PAULO

$2012 ; 24(1)$ :

$67-75$, JAN-ABR

\section{N T R O D U ÇÃ O}

O estudo das discrepâncias maxilares constitui na atualidade uma área de grande interesse científico na Odontologia.

A expansão rápida da maxila (ERM) consiste numa técnica eficiente e permanente na tentativa de compensar as relações maxilomandibulares deficientes sendo inicialmente descrita por Angell $^{1}$ (1860) e Haas² (1961). Possui como objetivo principal a disjunção maxilar através de expansores palatinos e, dessa forma, melhora a dimensão transversal dos pacientes acometidos pelas deficiências maxilares.

A partir do expansor ortodôntico dentomucosuportado de Haas, muitos outros foram desenvolvidos, incluindo o Haas modificado e o aparelho de Hyrax. Este último é um expansor dentosuportado que acarreta inclinações dentoalveolares posteriores maiores, se comparadas com o expansor Haas.

É importante salientar que a escolha de um disjuntor para a correção maxilar é problemática e pode justificar-se por aspectos importantes como a estrutura óssea, os processos alveolares, a presença ou não dos elementos dentais, a higienização do paciente, a dentição quanto a ser mista ou permanente, a necessidade de um aumento maior anterior ou posterior, as inclinações dentoalveolares, as distâncias interdentais e as desestabilizações verticais. Outro fator importante a ser levantado consiste na idade do paciente, devendo-se levar em conta a sua maturação óssea para definir a melhor técnica de expansão transversal maxilar.

A técnica de expansão rápida cirúrgica de maxila (ERMA) pode ser realizada através da osteotomia maxilar e da rafe palatina associada a um expansor palatino, ou osteotomia LeFort I associada com segmentações maxilares.

Não existe ainda um consenso sobre qual seja a melhor técnica de expansão maxilar ou mesmo qual o melhor expansor e, nesse caso, o objetivo deste estudo foi justamente tentar responder algumas das questões mais frequentes, baseando-se na revisão da literatura atual.

\section{REVISÃO DE LITERATURA}

Em casos de pacientes com deficiência severa, perdas ósseas alveolares e ausências dentárias posteriores, o expansor de Haas modificado é o mais adequado segundo (Biederman ${ }^{3}$ 1968).

Bishara e Staley ${ }^{4}$ (1987) afirmam que o insucesso da ERM não é proveniente somente, mas principalmente, da faixa etária, devido à consolidação óssea, como também pode estar correlacionado a fatores de risco como o tipo de expansor e o tempo de ativação.

A ERMA pode variar no que diz respeito às técnicas empregadas, que podem ser: (1) a osteotomia maxilar Lefort I, segmentação maxilar com reposicionamento lateral da partes para correção da atresia maxilar e (2) a osteotomia maxilar parcial realizada em conjunto com um dispositivo expansor. Assim, Betts et al..$^{5}$ (1995) apontaram a técnica parcial como a mais estável clinicamente, quando comparada à expansão segmentada e ainda preconizada para os casos clínicos com discrepância acentuada e inclinação vestibular de molares.

Asanza et al. ${ }^{6}$ (1997) utilizaram os expansores Haas e Hyrax num grupo de 14 pacientes e observaram um aumento na dimensão vertical no segundo grupo, porém as inclinações dentoalveolares e a expansão maxilar nos dois grupos não tiveram diferenças clínicas significativas.

Rabelo et al. (2002) afirmam que não existe uma concordância na literatura em relação a uma idade fixa estipulada para indicação da ERM.

Stuart e Wiltshire ${ }^{8}$ (2003) obtiveram $7 \mathrm{~mm}$ de expansão com $3 \mathrm{~mm}$ de diastema utilizando a técnica ERM com expansor Haas, num paciente de 19 anos do sexo masculino.

Oliveira et al. ${ }^{9}$ (2004) afirmaram que a cobertura em acrílico no expansor Haas dirigiria o vetor de força para o centro de resistência da maxila, permitindo o aumento da dimensão transversal, não afetando a dimensão sagital.

Davidovitch et al. ${ }^{10}$ (2005), em 28 pacientes entre 8 e 20 anos de idade, observaram maiores inclinações dentoalveolares em molares posteriores utilizando 
o expansor Hyrax quando comparado ao Haas.

Garib et al. ${ }^{11}$ (2005) utilizaram os expansores Haas e Hyrax em 11 meninas entre 11 e 14 anos de idade, divididas em dois grupos e não observaram, quanto à expansão maxilar transversal, diferenças representativas nos dois grupos; porém, a inclinação de pré-molares superiores mostrou-se superior nos expansores Hyrax.

Pastori et al. ${ }^{12}$ (2007) obtiveram resultados satisfatórios em seu estudo através da técnica de ERMA, onde realizaram uma osteotomia LeFort I, separando inclusive as lâminas pterigóideas e ativação do aparelho expansor Hyrax. Os autores afirmam que um tratamento de ERM deve levar em consideração a faixa etária do paciente, preconizando a idade máxima para mulheres até os 14 anos e 16 anos para os homens.

Segundo Provatidis et al..$^{13}$ (2006), as áreas de resistência ósseas no maxilar são: a sutura mediana palatina, a sutura frontal, a sutura zigomático-temporal, a intermaxilar e, principalmente, o processo pterigóide, onde alguns cirurgiões optam por não realizar a separação do mesmo, visto que o risco de dano ao plexo pterigóide é grande.

Num estudo comparativo com 31 pacientes, divididos aleatoriamente, onde o Grupo A utilizava o expansor Hyrax e o Grupo B o expansor Haas, Scanavini et al. ${ }^{14}$ (2006) constataram que os expansores Hyrax demonstraram maior risco de recidiva.

Claro et al. ${ }^{15}$ (2006) encontraram mudanças significativas nas distâncias intercaninos e intermolares utilizando o aparelho ortodôntico Hyrax, dessa forma comprovando um aumento transversal posterior maior.

Ribeiro et al. ${ }^{16}$ (2006), num estudo realizado em pacientes do sexo feminino com idade de 17 anos, obtiveram, através do expansor Haas modificado, um ganho de $6,5 \mathrm{~mm}$ entre os primeiros molares superiores, sem relatos de desconforto, dor ou edema durante o tratamento.

Vasconcelos et al. ${ }^{17}$ (2006) utilizaram 10 pacientes entre 18 e 40 anos. No Grupo A realizaram osteotomias LeFort I e separação da sutura intermaxilar e no Grupo B o mesmo procedimento com a separação dos processos pterigóides. Os autores concluíram que não houve diferenças significativas entre as duas técnicas, não justificando, portanto, a separação dos processos pterigóideos.

Malmström e Gurgel ${ }^{18}$ (2007) afirmam a ineficácia nas técnicas de ERM em pacientes adultos que já ultrapassaram o pico de crescimento.

Para Ferreira et al..$^{19}$ (2007), o expansor Hyrax é o mais indicado para a expansão rápida cirúrgica de maxila, dada a sua facilidade para higienização.

Holberg et al..$^{20}$ (2007), utilizando modelos virtuais em 3D, observaram que as osteotomias de maxila até a fossa pterigopalatina diminuem o estresse ósseo principalmente nos ossos da base do crânio e do terço médio da face na ERMA.

Segundo Brites ${ }^{21}$ (2007), a ERMA é uma técnica segura, de fácil execução e as intercorrências são geralmente eventuais. Além disso, os pacientes mostram-se satisfeitos.

De acordo com Scartezini et al. ${ }^{22}$ (2007), a opção pela melhor técnica cirúrgica de ERMA consiste na observação de alguns critérios clínicos: deficiências transversais superiores a $5 \mathrm{~mm}$; deformidades transversais isoladas; deformidades transversais associadas a deformidades verticais e/ou anteroposteriores e deficiências transversais da maxila com maior envolvimento na região anterior.

Chamberland e Proffit ${ }^{23}$ (2008) utilizaram a técnica de expansão rápida cirúrgica associada a um expansor maxilar Hyrax em 20 pacientes entre 15 e 54 anos, obtendo resultado final médio satisfatório.

Vidotti e Trindade ${ }^{24}$ (2008) observaram, em estudos com rinometria acústica, um aumento significativo no fluxo aéreo da cavidade nasal, devido às terapias de expansão transversal da maxila, isso devido ao aumento do volume anterior da cavidade nasal.

Ramires et al. ${ }^{25}$ (2008) afirmam que as deficiências transversais da maxila são deformidades dentofaciais relativamente comuns em crianças e adultos. Os pacientes nesta situação podem apresentar com
ALMEIDA TE SAAVEDRA J PAVLOVSKY M SCROCCO JA SANTOS MG MONTEIRO CG

EXPANSÃO RÁPIDA DA MAXILA NÃO

CIRÚRGICA E CIRÚRGICA: REVISÃO DE LITERATURA

\section{9}

REV, ODONTOL.

UNIV. CID, SÃo PAULO

2012; $24(1)$ : 67 75, JAN-ABR 
ALMEIDA TE

SAAVEDRA J

PAVLOVSKY M

SCROCCO JA

SANTOS MG

MONTEIRO CG

EXPANSÃO RÁPIDA

DA MAXILA NÃO

CIRÚRGICA E

CIRÚRGICA :

REVISÃO DE

LITERATURA

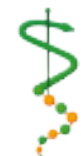

REV, ODONTOL.

UNIV, CID, SÃO

PAULO

2012; $24(1)$ :

$67-75$, JAN-ABR frequência mordida cruzada anterior, posterior, unilateral, bilateral; apinhamentos dentários; retrognatismo; prognatismo; dentre outros, incluindo-se a dificuldade respiratória ocasionada pela diminuição da distância entre as paredes laterais da cavidade nasal.

Haas $^{2}$ (1961) foi um dos pioneiros na técnica da ERM, desenvolvendo um aparelho disjuntor composto de bandas ortodônticas situadas no primeiro molar e primeiro pré-molar de cada lado, que são unidas por uma estrutura de fio de aço inox por vestibular e palatina das bandas, e um parafuso central. Estes são incorporados a um elemento acrílico em contato com a mucosa palatina, sendo, portanto, dentomucosuportados, e gerando uma força de tração na base óssea, sem a movimentação ortodôntica dentária, segundo (Almeida e Almeida ${ }^{26}$ 2008).

O parafuso expansor desses aparelhos ortodônticos tem o objetivo de romper a resistência óssea oferecida pela sutura maxilar, sendo esta, segundo Consolaro e Consolaro ${ }^{27}$ (2008), uma estrutura que desempenha um papel importante no crescimento craniomandibular e, ainda, a sua manipulação poderia corrigir as falhas ortopédicas. Além disso, afirmam que em adultos que atingiram a maturidade óssea, as técnicas de ERM são ineficazes. Esses autores indicam a técnica de expansão rápida cirurgicamente assistida sem a osteotomia dos processos pterigóides.

Neves et al. ${ }^{28}$ (2008) acreditam que a melhor época para a realização de procedimentos como a ERM é o período puberal, sendo mais fácil a correção até os 13 anos de idade, sem excluir os pacientes com até 18 anos.

Kilic et al. ${ }^{29}$ (2008) avaliaram a expansão dentoalveolar utilizando dois expansores maxilares em 39 pacientes. O primeiro grupo utilizou o aparelho expansor Hyrax, enquanto que o segundo grupo utilizou o Haas. Foram traçadas linhas com sulfato de bário entre os molares superiores nos modelos de estudo e analisados os ângulos de inclinação digitalmente através de um software. Os resultados obtidos demonstraram maior inclinação posterior de molares no primeiro grupo e, assim, os autores indicam os disjuntores de Haas.

Janson et al..$^{30}$ (2008) concluíram, então, que a técnica de segmentação da maxila possui grande vantagem em relação à quantidade de procedimentos operatórios, pois se realiza apenas com uma única intervenção cirúrgica, reduzindo a morbidade, o impacto psicológico do paciente e ainda reduz custos do tratamento.

Ho et al. ${ }^{31}$ (2008) realizaram a técnica cirúrgica por segmentação maxilar em 6 pacientes com média de idade de 11 anos e obtiveram $5,75 \mathrm{~mm}$ de expansão entre molares, 1,03 $\mathrm{mm}$ de aumento interdental entre molares e pré-molares, aumento de profundidade de $0,5 \mathrm{~mm}$, aumento do perímetro no arco dental e efeito vertical não significativo.

Rossi et al.32 (2009), em seu estudo retrospectivo, concluíram que a técnica ERM está contraindicada em casos de ausência dentária múltipla, grandes inclinações dentoalveolares para vestibular, recessão gengival, perda óssea alveolar, mobilidade dentária posterior e em pacientes adultos com maturação esquelética avançada.

Para Magnusson et al. ${ }^{33}$ (2009), uma osteotomia menos invasiva é mais eficiente para o pós-operatório, diminuindo os riscos associados à terapia da ERMA, e no seu estudo com 26 pacientes foram realizadas osteotomias bilaterais a partir da abertura piriforme até a fissura pterigóidea, porém sem a osteotomia palatina.

Lagravere et al. ${ }^{34}$ (2010) utilizaram os aparelhos Hyrax e Haas em 62 pacientes que necessitavam de expansão rápida da maxila e observaram maior expansão ao nível das coroas dos primeiros molares e pré-molares superiores nos aparelhos Hyrax.

Wang et al. ${ }^{35}$ (2010), num estudo comparativo realizado com 18 pacientes divididos em dois grupos um com tratamento ortodôntico pré-cirúrgico e outro não, observaram que as dimensões transversais pós-cirúrgicas foram semelhantes nos dois grupos, sendo discutível a utilização do tratamento pré-ortodôntico para ERMA.

Canuto et al. ${ }^{36}$ (2010) observaram, num estudo comparativo, 48 pacientes portadores de classe I e II, divididos em dois grupos, um realizando a ERM e o outro 
utilizando somente aparelhos fixos sem a técnica ERM. Concluíram que a técnica ERM mostrou-se superior, aumentando satisfatoriamente a dimensão transversal sem alterar a estabilidade maxilar anterior e, principalmente, o alinhamento dos dentes anteriores.

\section{I S C U S S ÃO}

As deficiências transversais devem passar por uma análise minuciosa por meio de exame clínico, análise dos modelos de gesso, radiografias cefalométricas e oclusais na tentativa de visualizar alterações prevalentes como mordidas cruzadas anteriores, posteriores, inclinações dentoalveolares, falta de elementos dentários, prognatismo ou retrognatismo, recessão gengival, perda óssea alveolar, mobilidade dentária posterior e maturação esquelética, para se selecionar a técnica adequada de expansão maxilar.

\section{Indicação da Expansão Rápida de Maxila (ERM)}

Com relação à indicação, a técnica de ERM é adequada para pacientes com maturação óssea maxilar incompleta e deficiência transversal de maxila que não possa ser corrigida por aparelho fixo (Canuto et al. $\left.{ }^{36}, 2010\right)$.

Outro benefício indireto das técnicas de expansão de maxila é a melhora do fluxo aéreo superior diminuindo as dificuldades respiratórias, de acordo com Ramires et al. ${ }^{25}$ (2008), já que promove o aumento da cavidade aérea (Vidotti e Trindade ${ }^{24}$, 2008).

Assim, a idade mais aceita para a realização da ERM varia muito segundo a literatura, sendo proposta para pacientes com faixa etária máxima de 14 anos de idade em mulheres e 16 anos nos homens, segundo (Pastori et al. ${ }^{12}$ 2007). Já Neves et al. ${ }^{28}$ (2008) observaram maiores facilidades em pacientes na fase puberal até 13 anos de idade, porém não excluindo aqueles com até 18 anos, devido à não consolidação óssea.

Por outro lado, Chamberland e Proffit ${ }^{23}$ (2008) tiveram sucesso com a faixa etária entre 15 e 54 anos, já Ribeiro et al. ${ }^{16}$ (2006) em uma paciente de 17 anos e Stu- art e Wiltshire ${ }^{8}$ (2003) obtiveram resultados promissores, utilizando a técnica ERM com um expansor Haas num paciente de 19 anos de idade, contradizendo os autores que afirmam a eficácia da técnica somente em pacientes com maturação óssea incompleta.

Percebe-se, então, que não existe uma concordância na literatura em relação a uma idade fixa para a indicação da ERM, de acordo com Rabelo et al. ${ }^{7}$ (2002). Isso porque existe uma ineficácia das técnicas de ERM em pacientes adultos que já ultrapassaram o pico de crescimento, segundo Malmström e Gurgel ${ }^{18}$ (2007).

Além da consolidação óssea como fator limitante, Rossi et al. ${ }^{32}$ (2009) afirmam que a técnica ERM está contraindicada em casos de ausência dentária múltipla, grandes inclinações dentoalveolares para vestibular, recessão gengival, perda óssea alveolar, mobilidade dentária posterior e em pacientes adultos com maturação esquelética avançada.

Já com relação ao insucesso da ERM, este não é proveniente somente da faixa etária devido à consolidação óssea, como pode também estar correlacionado a fatores de risco como tipo de expansor e o tempo de ativação (Bishara e Staley ${ }^{4}$, 1987).

\section{Indicação da Expansão Rápida Ci- rúrgica de Maxila (ERMA)}

Para os pacientes com idade mais avançada e consequente maturação óssea completa, recomendar-se-ia a expansão maxilar cirurgicamente assistida (ERMA), segundo os autores Consolaro e Consolaro $^{27}$ (2008) e Rossi et al. ${ }^{32}$ (2009). Além dessa indicação, a ERMA também está indicada, segundo Scartezini et al. ${ }^{22}$ (2007), para deficiências transversais superiores a $5 \mathrm{~mm}$, deformidades transversais isoladas, deformidades transversais associadas a deformidades verticais e/ou anteroposteriores e em deficiências transversais da maxila com maior envolvimento na região anterior.

\section{Seleção do Expansor Ideal}

Embora seja difícil a seleção de um expansor ideal, Ferreira et al. ${ }^{19}$ (2007) pre-
ALMEIDA TE SAAVEDRA J PAVLOVSKY M SCROCCO JA SANTOS MG MONTEIRO CG

EXPANSÃO RÁPIDA DA MAXILA NÃO

CIRÚRGICA E CIRÚRGICA: REVISÃo DE LITERATURA

\section{1}

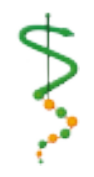

REV, ODONTOL.

UnIV. CID. SÃo

PAULO

2012; $24(1)$ : 67 -

75, JAN-ABR 
ALMEIDA TE

SAAVEDRA J

PAVLOVSKY M

SCROCCO JA

SANTOS MG

MONTEIRO CG

EXPANSÃO RÁPIDA

DA MAXILA NÃO

CIRÚRGICA E

CIRÚRGICA :

REVISÃO DE

LITERATURA

REV, ODONTOL.

UNIV, CID, SÃO

PAULO

$2012 ; 24(1)$ :

$67-75$, JAN-ABR
I SSN 1983-5183

conizam a utilização do expansor Hyrax, devido à sua facilidade de higienização, tanto na ERM como na ERMA. Por outro lado, Biederman ${ }^{3}$ (1968) indicou o aparelho de Haas modificado em pacientes com perdas ósseas alveolares e ausências dentárias posteriores.

Deve-se pensar que o disjuntor Haas modificado produz uma inclinação dentoalveolar menor que os aparelhos Hyrax, além de aumentar a distância interdental entre os caninos. Também a cobertura em acrílico, no expansor Haas, dirige o vetor de força para o centro de resistência da maxila, aumentando a dimensão transversal, não afetando a dimensão sagital, segundo Oliveira et al. ${ }^{9}$ (2004), além de não provocar movimentação ortodôntica dentária Almeida e Almeida ${ }^{26}$ (2008). Já os aparelhos Hyrax possuem um maior aumento interdental entre os molares e pré-molares e um aumento transversal posterior, de acordo com Garib et al. ${ }^{11}$ (2005), Davidovitch et al. ${ }^{10}$ (2005), Claro et al. ${ }^{15}$ (2006) e Lagravere et al. ${ }^{34}$ (2010).

Já Asanza et al. ${ }^{6}$ (1997) não observaram diferenças clínicas significativas entre Haas e Hyrax. Embora a expansão maxilar seja superior nos disjuntores Hyrax, segundo Kilic et al..$^{29}$ (2008), a recomendação deste estudo, impera para a utilização dos disjuntores de Haas modificado, pois este direciona sua força de tração para o centro da maxila, não afetando tanto a inclinação dentária.

Por fim, a literatura afirma que a utilização dos disjuntores de Hyrax apresentam um maior risco de recidiva quando comparados com os de Haas, segundo Scanavini et al. ${ }^{14}$ (2006).

\section{Seleção da Técnica de ERMA}

Magnusson et al. ${ }^{33}$ (2009) indicaram a não realização da osteotomia da rafe palatina para reduzir o risco de sangramento operatório e complicações pós-operatórias como epistaxe, infecções sinusais, necroses avasculares, ulcerações, dor, edema, reabsorção radicular, perda de órgão dental, extrusões dentárias, desvitalizações e hemorragias. Em contrapartida, Brites ${ }^{21}$ (2007) afirmou que as intercorrências são geralmente eventuais, não havendo números significativos de casos na literatura, pois a técnica da ERMA é de fácil execução.

Outro aspecto a ser discutido na ERMA refere-se à separação dos pilares pterigóideos. Embora Provatidis et al. ${ }^{13}$ (2006), Vasconcelos et al. ${ }^{17}$ (2006) e Consolaro e Consolaro $^{27}$ (2008) preconizem a não realização dessa osteotomia devido a danos ao plexo pterigóideo, por outro lado Holberg et al. ${ }^{20}$ (2007) evidenciam a importância da mesma, devido à diminuição do estresse ósseo, principalmente nos ossos da base do crânio e do terço médio da face.

Por fim, a literatura afirma que a utilização de aparelho ortodôntico pré-cirurgico não melhorou as dimensões transversais pós-cirurgicas, segundo Wang et al. ${ }^{35}$ (2010).

Quando se compara a técnica de segmentação maxilar com a ERMA percebe-se que a literatura afirma que a técnica segmentada demonstrou elevadas taxas de expansão maxilar (Ho et al. ${ }^{31}$ 2008). Outra vantagem da técnica segmentada é a realização de uma única intervenção cirúrgica, com isso reduzindo-se o período de convalescença, os custos e o impacto psicológico do paciente, de acordo com Janson et al. ${ }^{30}$ (2008). Mas, por outro lado, a ERMA é mais estável clinicamente quando comparada à expansão segmentada sendo, ainda, preconizada para os casos clínicos com discrepância acentuada e inclinação vestibular de molares (Betts et al. $\left.{ }^{5}, 1995\right)$.

\section{CONCLUSÃO}

Após análise e discussão da revisão literária, é possível concluir que:

- A ERM está indicada para pacientes com maturação óssea incompleta, não existindo um consenso na literatura quanto à melhor idade para o tratamento.

- A ERMA está indicada para: maturação óssea completa, deficiências transversais superiores a $5 \mathrm{~mm}$, deformidades transversais isoladas, deformidades transversais associadas a deformidades verticais e/ou anteroposteriores e em deficiências transversais da maxila com maior envolvimento na região anterior.

- O disjuntores de Hyrax apresentam melhor higienização, sendo indica- 
dos para ERMA, já os de Haas apresentam melhor distribuição das forças, pois possuem uma cobertura de acrílico que se apoia sobre o palato.

- Tanto a ERM quanto a ERMA melhoram o fluxo aéreo superior, diminuindo as dificuldades respiratórias, já que promovem o aumento da cavidade aérea.
- Não existe melhora significativa nos resultados da expansão quando se realiza a osteotomia do pilar pterigoide.

- A ERMA é mais estável clinicamente quando comparada à expansão segmentada sendo, ainda, preconizada para os casos clínicos com discrepância acentuada e inclinação vestibular de molares.

\section{REFERÊNCIAS}

1. Angell E. Treatment of irregularity of the permanent or adult teeth. Part. 2. Dental Cosmos 1860 Jun.;1(10):599-600.

2. Haas A. Rapid expansion of the maxillary dental arch and nasal cavity by opening the mid-palatal suture. Angle Orthod Appleton 1961 Apr;31(2):73-90.

3. Biederman W. A hygienic appliance for rapid expansion. JPO J Pract Orthod 1968 Feb;2(2):67-70.

4. Bishara SE, Staley RN. Maxillary expansion: clinical implications. Am J Orthod Dentofacial Orthop 1987 Jan;91(1):3-14.

5. Betts NJ, Vanarsdall RL, Barber HD, Higgins-Barber K, Fonseca RJ. Diagnosis and treatment of transverse maxillary deficiency. Int J Adult Orthodon Orthognath Surg 1995 10(2):75-96.

6. Asanza S, Cisneros GJ, Nieberg LG. Comparison of Hyrax and bonded expansion appliances. Angle Orthod 1997 67(1):15-22.

7. Rabelo L, Bastos E, Germano A, Passeri L. Expansão de maxila cirurgicamente assistida sob anestesia local. Rev Dent Press Ortodon Ortop Facial 2002 jan/fev.;7(1):739.

8. Stuart DA, Wiltshire WA. Rapid palatal expansion in the young adult: time for a paradigm shift? J Can Dent Assoc 2003 Jun;69(6):374-7.

9. Oliveira NL, Da Silveira AC, Kusnoto B, Viana G. Three-dimensional assessment of morphologic changes of the maxilla: a comparison of 2 kinds of palatal expanders. Am J Orthod Dentofacial Orthop 2004 Sep;126(3):354-62.

10. Davidovitch M, Efstathiou S, Sarne O, Vardimon AD. Skeletal and dental response to rapid maxillary expansion with 2 - versus 4-band appliances. Am J Orthod Dentofacial Orthop 2005 Apr;127(4):483-92.

11. Garib DG, Henriques JF, Janson G, Freitas MR, Coelho RA. Rapid maxillary expansion--tooth tissue-borne versus tooth-borne expanders: a computed tomography evaluation of dentoskeletal effects. Angle Orthod 2005 Jul;75(4):548-57.

12. Pastori C, Marzola C, Toledo-Filho JF, Azenha M, Cavalieri-Pereira L. Expansão rápida da maxila cirurgicamente assistida - Revista da literatura, técnica cirúrgica e relato de caso. Revista de odontologia [serial on the Internet]. 2007; 7: Available from: http://www.actiradentes.com.br/revista/2007/textos/42RevistaATO-Expansao_Maxila.pdf.

13. Provatidis C, Georgiopoulos B, Kotinas A, MacDonald JP. In vitro validated finite element method model for a human skull and related craniofacial effects during rapid maxillary expansion. Proc Inst Mech Eng H 2006 Nov;220(8):897-907.

ALMEIDA TE SAAVEDRA J PAVLOVSKY M SCROCCO JA santos MG MONTEIRO CG

EXPANSÃO RÁPIDA DA MAXILA NÃO

CIRÚRGICA E

CIRÚRGICA :

REVISÃo DE

LITERATURA
Rev. OdOntOl.

UnIV. CID. São

PAulo

2012; $24(1): 67$.

75, JAN-ABR 
ALMEIDA TE

SAAVEDRA J

PAVLOVSKY M

SCROCCO JA

SANTOS MG

MONTEIRO CG

EXPANSÃO RÁPIDA

DA MAXILA NÃO

CIRÚRGICA E

CIRÚRGICA :

REVISÃO DE

LITERATURA

74

REV, ODONTOL.

UNIV. CID. SÃO

PAULO

2012; 24(1):

$67-75, J A N-A B R$

14. Scanavini MA, Reis SAB, Simões MM, Gonçalves RAR. Avaliação comparativa dos efeitos maxilares da expansão rápida da maxila com os aparelhos de Haas e Hyrax. Revista Dental Press de Ortodontia e Ortopedia Facial 2006 11(1):60-71.

15. Claro C, Abrão J, Reis S, Fantini S. Correlation between transverse expansion and increase in the upper arch perimeter after rapid maxillary expansion. Braz Oral Res 2006 jan./mar.;20(1):76-81.

16. Ribeiro G, Vieira G, Ritter D, Tanaka O, Weissheimer A. Expansão maxilar rápida não cirúrgica em paciente adulto. Uma alternativa possível. Rev Clín Ortodon Dental Press 2006 abr./maio;5(2):70-7.

17. Vasconcelos BCdE, Caubi AF, Dias E, Lago CA, Porto GG. Expansão rápida da maxila cirurgicamente assistida: estudo preliminar. Rev Bras Otorrinolaringologia 2006 72(457-61.

18. Malmström MFV, Gurgel JdA. Avaliação da neoformação óssea na sutura palatina mediana por meio de radiografia digitalizada após a expansão assistida cirurgicamente. Rev Dent Press de Ortodontia e Ortopedia Facial 2007 12(82-93.

19. Ferreira CMP, Ursi W, Atta JY, Lyra MCO, Lyra FA. Efeitos dentais e esqueletais mediatos da E.R.M. utilizando o disjuntor Hyrax. Revista Dental Press de Ortodontia e Ortopedia Facial 2007 12(36-48.

20. Holberg C, Steinhauser S, Rudzki-Janson I. Rapid maxillary expansion in adults: cranial stress reduction depending on the extent of surgery. Eur J Orthod 2007 Feb;29(1):31-6.

21. Brites F. A study of complications with surgical assisted rapid maxillary expansion. Rev Bras Cirurgia e Traumatologia Buco-Maxilo-Facial 2007 1(101-8.

22. Scartezini G, Saska S, Dantas J, Hochuli-Vieira E, Gabrielli M. Expansão cirúrgica da maxila em pacientes adultos: expansão rápida assistida cirurgicamente ou osteotomia Le Fort I segmentar. Revista de Odontologia da UNESP 2007 267-273;36(3):

23. Chamberland S, Proffit WR. Closer look at the stability of surgically assisted rapid palatal expansion. J Oral Maxillofac Surg 2008 Sep;66(9):1895-900.

24. Vidotti BA, Trindade IEK. Os efeitos da expansão rápida da maxila sobre a permeabilidade nasal avaliados por rinomanometria e rinometria acústica. Rev Dental Press de Ortodontia e Ortopedia Facial 2008 13(59-65.

25. Ramires T, Maia RA, Barone JR. Alterações da cavidade nasal e do padrão respiratório após expansão maxilar. Rev Bras Otorrinolaringologia 2008 74(763-9.

26. Almeida R, Almeida M. Aparelho disjuntor fusionado, uma nova visão da disjunção palatina. Ortodontia SPO 2008 41(1):55-9.

27. Consolaro A, Consolaro MFM-O. Expansão Rápida da Maxila e Constrição Alternadas (ERMC-Alt) e técnica de Protração Maxilar Ortopédica Efetiva: extrapolação de conhecimentos prévios para fundamentação biológica. Revista Dental Press de Ortodontia e Ortopedia Facial 2008 13(18-23.

28. Neves M, Itaborahy W, Pacheco M. Associação entre a Ortopedia Funcional e Ortodontia para o tratamento de Mordida cruzada com assimetria facial - relato de caso clínico. Rev Clín Ortodon Dental Press 2008 fev./mar.;7(1):80-6.

29. Kilic N, Kiki A, Oktay H. A comparison of dentoalveolar inclination treated by two palatal expanders. Eur J Orthod 2008 Feb;30(1):67-72.

30. Janson M, Janson G, Sant'Ana E, Nakamura A, de Freitas MR. Segmental LeFort I osteotomy for treatment of a Class III malocclusion with temporomandibular disorder. J Appl Oral Sci 2008 Jul-Aug;16(4):302-9. 
31. Ho CT, Lo LJ, Liou EJ, Huang CS. Dental and skeletal changes following surgically assisted rapid maxillary anterior-posterior expansion. Chang Gung Med J 2008 Jul-Aug;31(4):346-57.

32. Rossi RRP, Araújo MTd, Bolognese AM. Expansão maxilar em adultos e adolescentes com maturação esquelética avançada. Revista Dental Press de Ortodontia e Ortopedia Facial 2009 14(43-52.

33. Magnusson A, Bjerklin K, Nilsson P, Marcusson A. Surgically assisted rapid maxillary expansion: long-term stability. Eur J Orthod 2009 Apr;31 (2):142-9.

34. Lagravere MO, Carey J, Heo G, Toogood RW, Major PW. Transverse, vertical, and anteroposterior changes from bone-anchored maxillary expansion vs traditional rapid maxillary expansion: a randomized clinical trial. Am J Orthod Dentofacial Orthop 2010 Mar;137(3):304 e1-12; discussion -5.

35. Wang YC, Ko EW, Huang CS, Chen YR, Takano-Yamamoto T. Comparison of transverse dimensional changes in surgical skeletal Class III patients with and without presurgical orthodontics. J Oral Maxillofac Surg 2010 Aug;68(8):1807-12.

36. Canuto LF, de Freitas MR, Janson G, de Freitas KM, Martins PP. Influence of rapid palatal expansion on maxillary incisor alignment stability. Am J Orthod Dentofacial Orthop 2010 Feb;137(2):164 e1-6; discussion -5.

Recebido em: 24/03/2011

Aceito em: 28/03/2011

ALMEIDA TE

SAAVEDRA J

PAVLOVSKY M

SCROCCO JA

SANTOS MG

MONTEIRO CG

EXPANSÃO RÁPIDA

DA MAXILA NÃO

CIRÚRGICA E

CIRÚRGICA :

REVISÃO DE

LITERATURA 